\title{
Optimization of Modern Tram Operation Line Based on Genetic Algorithm
}

\author{
Yanhui $\mathrm{Li}^{1, \mathrm{a}}$, Kuanmin Chen ${ }^{2, \mathrm{~b}}$ \\ ${ }^{1}$ School of Highway, Chang'an University, Xi'an 710064, Shaanxi, China \\ ${ }^{2}$ School of Highway, Chang'an University, Xi'an 710064, Shaanxi, China

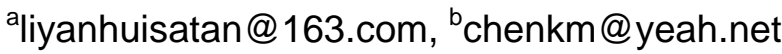

Keywords: Modern tram, Maximum flow, Genetic algorithm.

\begin{abstract}
The modern tram has both the attributes of rail transit and bus. Based on the point-line-plane planning method in public transportation and urban rail transit network planning, this study considers the tram line Network, the characteristics of the line, including the length of the line, the node choosing, the scale; in determining the boundary conditions under the premise, by gradually select the maximum flow, sub-maximum flow as the operating path, the genetic algorithm is used to analyze the optimization model. Through the actual analysis, the optimization model has a good effect in the planning and application of modern tram lines.
\end{abstract}

\section{Introduction}

The modern tram is a kind of medium-low volume of urban rail transit. The vehicle track is mainly laid on the road surface of the city. The vehicle is operated with other ground transportation. The passenger flow section is in the range of 0.6-1 million people / h, by running speed of $15-25 \mathrm{~km} / \mathrm{h}$. The low volume is suitable for the ground (independent road right), mixed transportation or elevated road. The layout of the station may take into account the one-sided layout on the sidewalk on either side of the street or the central layout on the central separation zone[1].

The tram system is more com-plex, higher cost, and the tram train is constitute of 2-7 modules[2]. With the development of urbanization, the tram have a further improvement for traffic volume. So the tram has very broad applicability. The typical volume of trams are shown in Table 1[3].

Table 1 Capacity of some Citadis tramways of Alstom

\begin{tabular}{|c|c|c|c|c|c|c|c|}
\hline \multirow{2}{*}{ Mode } & \multirow{2}{*}{ Length(m) } & \multirow{2}{*}{ Width(m) } & \multirow{2}{*}{$\begin{array}{c}\text { Capacity /Seats } \\
\text { (person) }\end{array}$} & \multicolumn{3}{|c|}{ Capacity at different operating intervals (person /h) } \\
\cline { 5 - 7 } & & & $6 \mathrm{~min}$ & $5 \mathrm{~min}$ & $4 \mathrm{~min}$ & $3 \mathrm{~min}$ \\
\hline 1 & 22 & 2.65 & $145 / 40$ & 1450 & 1740 & 2175 & 2900 \\
\hline 2 & 32 & 2.65 & $230 / 64$ & 2300 & 2760 & 3450 & 4600 \\
\hline 3 & 44 & 2.65 & $300 / 78$ & 3000 & 3600 & 4500 & 6000 \\
\hline 4 & $2 \times 32$ & 2.65 & $460 / 128$ & 4600 & 5520 & 6900 & 9200 \\
\hline 5 & $2 \times 44$ & 2.65 & $600 / 156$ & 6000 & 7200 & 9000 & 12000 \\
\hline
\end{tabular}

At present, most of the tram line planning is similar to urban rail transit network planning method. Because of the difference of tram and urban rail transit and the characteristics of conventional bus ,on the basis of the characteristics of tram, through the improvement of the point-line-plane method of rail transit network planning, then obtains the optimization method of operating planning which adapts to the characteristics of tram.

\section{Model description}

\subsection{Basic technical line of railway network planning •}

The most important part of urban rail transit network planning is point - line - plane analysis process. "Point" is a kind of passenger distribution center, mainly including sub-centers, regional centers at all levels, transport hubs at all levels, commercial centers, residential areas, industrial groups, tourist attractions, etc. Based on these passenger distribution points, the line between the distribution points and other areas are necessary to adjust the direction of the passenger trip, thus 
forming the basic form of the railway network; "line" is the traffic corridor, represents the network line of the possible path.We can analysis the main road traffic characteristics, especially transit operation characteristics and the possible impact of rail transit, including the status quo, planning conditions, the main project difficult, along with the land use nature, line function, the impact on the overall network; "surface" is the overall form of the area ,it's the basic structure of the proposed network, including urban structure, land use, population and employment, social and economic development level, large-scale projects, environment, cultural protection, traffic development strategy, road network structure, traffic structure and other characteristics, hub layout, bus network, rail network concept, future traffic macro analysis[4].

\subsection{Optimization ideas .}

1.Node choosing: According to the characteristics of the first and final stations, and combine the feasible nodes.In this process, consider the importance of relatively large node pairs as much as possible, regardless of all node pairs. By checking the scale, transportation efficiency and other constraints, make sure the chosen node relatively reasonable.

2.In the feasible line pairs, a number of better-performing lines are selected as viable lines of the feasible endpoints to form a feasible subset of the lines.Each feasible line could be selected from the feasible route subset then establish a number of feasible lines. At the same time of choosing feasible nodes in the best line, but also consider some of the better lines.

3.Optimization: Establishing the optimization model to determine whether the program to meet other constraints, whether the objective function could achieve the optimistic function better. If satisfied, output the program as the final program directly . Otherwise, according to the intelligent optimization algorithm to recalculate a new program, and then to determine the output until the requirements meet.[5]

\subsection{Optimization ideas .}

The choice of node pairs is actually the choice of the first station and the final station of the tram, which must meet the basic requirements of the tram line at the end of the station. A feasible node is a node that can be used to set up the first and last stations of a tram network. In addition to requiring a large demand for trams, it is necessary to meet the basic requirements of land use. Assume that the feasible node is represented as $v_{i}(i=1,2, \cdots)$. The feasible node pair refers to the pairing of the feasible nodes, and there is a path available between the two nodes that need to be paired, and the distance should be appropriate. Assume that the feasible node pair is expressed as $B=\left\{\left(v_{i}, v_{j}\right)^{v} \mid v=\right.$ $\left.1,2, \cdots, n_{2}\right\}$, and $n_{1} \leqslant n_{2}$. Therefore ,a feasible node pair is a set of a number of feasible node pairs.

1. Pre-selections of node pairs

This should meet the line length requirements.

$$
l_{\min } \leqslant \Gamma_{i j} \leqslant l_{\max }
$$

$\Gamma_{i j}$ is the minimum effective path length between station $v_{i}$ and station $v_{j}, l_{\min }$ is the limitation of minimum line length. $l_{\max }$ is the limitation of maximum line length. These two limitations will also satisfy the feasible node pair of the above formula as a pre-selected feasible node pair meantime. After the pre-selection of the point set, it is optimized from the point to be selected.

2. Choosing of feasible node pairs of trams

The importance of feasible node $M_{i j}$ is an indicator that describes the importance of a feasible node of the network. It is related to the location of the node and the demand for the rail transit OD, so make $M_{i j}=\operatorname{od}_{i j} Z_{i} Z_{j} \quad i, j=1,2, \cdots, n$, among them $Z_{i}$ is the location importance of the i-th node. $Z_{i}=\frac{1}{D_{i}} / \sum_{i=1}^{n} \frac{1}{D_{i}} \quad i=1,2, \cdots, n$, (similar to $Z_{j}$ ). 
$D_{i}$ is the sum of the distance from node $\mathrm{i}$ to all other nodes, $D_{i}=\sum_{j=1}^{n} d_{i j} \quad i=1,2, \cdots, n$ ( similar to $D_{j}$ ). The process of screening is to select $n_{2}$ significant degree of feasible nodes $n_{1}$ in a pre-selected node pair B.

3. The network size calculation of tramway

Combined with the characteristics of the tramway, the size of the network could be divided into two parts:[6]

(1)The method of traffic demand

$$
L=\frac{Q \alpha \beta \varphi}{\gamma}
$$

$L$ - Total network size,km; $Q$ - Total amount of daily travel, in 10 thousand ; $\alpha$ - Traffic sharing ratio of tramway ; $\beta$ - The railway accounts for the proportion of public transit ; $\varphi$ - Transfer factor; $\gamma$ - Average load intensity of line network, 10 thousand person / km.

(2) Network connectivity measurement method

$$
L=\sum_{i=1} \theta_{i} \lambda_{i} C_{i} \times \sqrt{N_{i} \times S_{i}}
$$

$\theta_{i}$ - The average extension coefficient of zone line $i$, straight when 1.0, but bending increases; $\lambda_{i}$ Level of economic development; $C_{i}$ - $i$ area connectivity coefficient, tree connection 1.0, network connection 2.0, triangle 3.22; $N_{i}$-The nodes number of zone $i ; S_{i}$-The area of zone $i$.

4. Determination of line set

(1)Search "the larger passenger flow " path. Find the first flow path, the second flow path, the third flow path. (2) Select $\left(v_{i}, v_{j}\right)^{1}, v=1,2, \cdots, n_{2}$ from B in turn, repeat the first step to find the second traffic path. Until the size of the network to achieve the size of the constraints, a node will be a combination of a number of lines into a subset, known as the subset of the nodes. (3) Calculate the transport efficiency of $k$ shortest path. The length of the $m$-th short path is $\Omega_{m}$, the OD of the $m$-th short path of tramway is $q_{m}$. Then, the transport efficiency of the $m$-th path is $\Omega_{m} / q_{m}$. According to the size of the transport efficiency in descending order, the lines arranged in the $\mu$-th are recorded as $r_{\mu}^{v}(\mu=1,2, \cdots, U)$. Place a total of $U$ lines placed in the front $\mathrm{U}$ into the feasible line subset $\varphi^{v}$.[7]

\section{Model establishment}

1. Objective function

(1)The transport efficiency of main network

The direct transport efficiency of the main network is defined as the ratio of the direct traffic between the bus and the total length of the bus line.

$$
\max \left\{\eta=Q_{\text {骨 }} / L\right\}
$$

$L$ - The total length of the main network; $Q_{m}$-The direct passenger volume of main network .

(2) The direct rate of main network

The direct rate of the skeleton line is defined as the proportion of the direct traffic to the total traffic. It reflects the entire network of direct service levels.

$$
\max \left\{\theta=Q_{m} / Q\right\}
$$

$Q_{m}$-The direct passenger volume of main network . $Q$ - Total bus demand.

2. Restrictions

(1) Length constraint

$$
R_{h} \leqslant l_{v}=\sum_{s=1}^{m_{v}-1} d_{r_{v s} r_{v(s+1)}} \leqslant 2 R_{h}
$$


$l_{v}$ represents the $v$-th line length of main network; $R_{h}$ is city radius; $d_{r_{v s} r_{v(s+1)}}$ is OD passenger flow on the $v$-th line $r_{v}$ from node $s$ to node $m_{v}$.

(2) Non - linear coefficient constraint

$$
1 \leqslant \eta_{v}=\frac{\sum_{s=1}^{m_{v}-1} d_{r_{v s} r_{v(s+1)}}}{\bar{d}_{r_{v 1} r_{k m_{v}}}} \leqslant 1.4
$$

$\eta_{v}$ is the non - linear coefficient of the $v$-th line ,it's no more than $1.4 ; \bar{d}_{r_{v 1} r_{k m_{v}}}$ is the minimum effective path length of $v$-th line .

(3) Line network density constraint

$$
\rho_{\min } \leqslant \rho=\frac{\sum_{v=1}^{n_{3}} l_{v}}{\omega \cdot \pi R_{h}^{2}} \leqslant \rho_{\max }
$$

$\rho$ is the main network density ; $\omega$ is the line repeating coefficient;

(4) Transfer constraint of the reachability no more than once

$$
\delta=\frac{\sum_{j}^{n} \sum_{i}^{n} o d_{i j} \cdot\left(m_{i j} \vee h_{i j}^{(1)}\right)}{\sum_{j}^{n} \sum_{i}^{n} o d_{i j} \cdot p_{i j}} \geqslant \delta_{\text {min }}
$$

$\delta$ is the transfer times of the reachability no more than once; $m_{i j} \vee h_{i j}^{(1)}$ decided by the direct matrix $M=\left(m_{i j}\right)_{n \times n}$ and the once transfer matrix $H^{1}=\left(h_{i j}^{(1)}\right)_{n \times n} ; p_{i j}$ is decided by the direct matrix $P=\left(p_{i j}\right)_{n \times n} ; \delta_{\text {min }}$ it the planning target , 70\%; od $d_{i j}$ is the OD demand of node $v_{i}$ and node $v_{j}$ of tramway network .

\section{Model solutions}

This model is belong to the optimal model, and it could be solved by genetic algorithm (GA) . GA is a simulation of natural biological evolution process of self-organization and adaptive artificial intelli-gence technology. It's based on the natural selection and genetic theory, the process combined the bio-logical evolution which the fittest ones survive and of random information exchange mechanisms with-in the group of chromosomes. Before the search, encoding the variables in some form (variable cod-ed called chromosomes), different chromosomes constitute a group. A new generation of groups is based on the following two steps: first, according to the fitness value of the chromosome then select the appropriate number of copies to be retained; Sec-ondly, process the selected chromosome with re-combination, mutation to generate new chromo-somes.[8]

The basic algorithm process is as follows:

(1). Selects $\mathrm{N}$ initial points randomly (described as a group, each point is called an individual), $X_{k}^{1}, X_{k}^{2}, \cdots, X_{k}^{n}, k=0$. (2) Calculate for each individual's fitness, $f\left(X_{k}^{i}\right), i=1,2, \cdots, N$.(3) Selection : Select $X_{k}^{1}, X_{k}^{\prime 2}, \cdots, X_{k}^{\prime n}$ from $X_{k}^{1}, X_{k}^{2}, \cdots, X_{k}^{n}$, and every $X_{k}^{j}(j=1,2, \cdots, N)$, probability of being selected is $P\left(X_{k}^{\prime i}=X_{k}^{j} \mid X_{k}^{1}, \cdots, X_{k}^{N}\right)=\frac{f\left(X_{k}^{j}\right)}{\sum_{l=1}^{N} f\left(X_{k}^{l}\right)}$.(4) Crossover : Select two individuals with the same probability from $X_{k}^{\prime 1}, X_{k}^{\prime 2}, \cdots, X_{k}^{\prime n}$, Take these two individuals with restructuring op-eration of given probability $P_{c}$ to produce two new individuals. Repeating this process until a new group $X_{k}^{\text {"1 }}, X_{k}^{\text {"2 }}, \cdots, X_{k}^{n}$ figure out .(5) Mutation : Change the value for each individual based on certain mutation rate $P_{m}$ randomly, and calculating a new generation of community.(6) Inspect the rationality 
of stop criteria, if the criteria meet the computational requirements and accuracy then operation is stopped, otherwise make $k+1=k$ and calculate it again .

\section{Case analysis}

The modern tram network has been planned nine lines in XiXian New Area, which contains two branch lines. The total length of network is 199 kilometers, and 150 stations, including 19 transfer sta-tions, 15 transfer stations between rail and subway . The four-stage model is used to assign the passenger flow with the use of Transcad software ,the result is shown in Figure 1. Simplify the prediction results of the network which shown as the Figure 2.

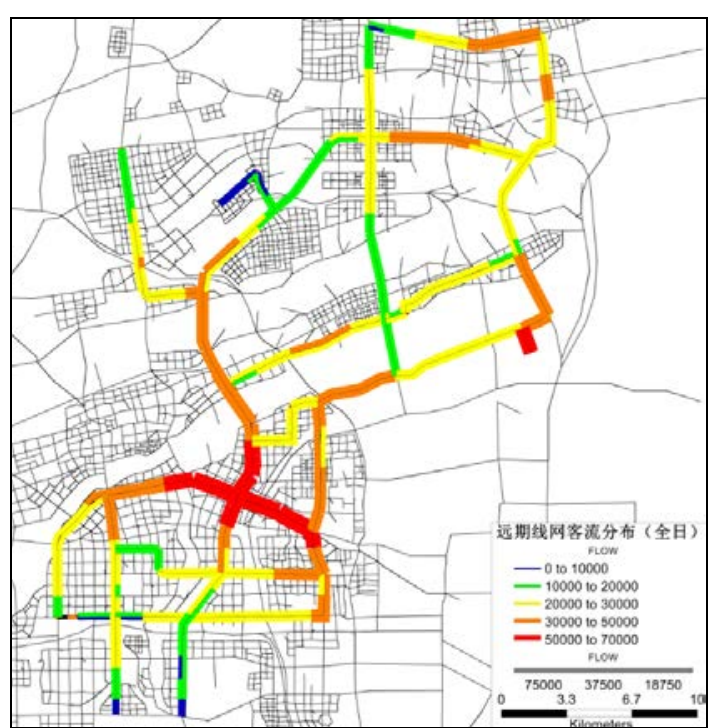

Fig .1 Tramway flow prediction results

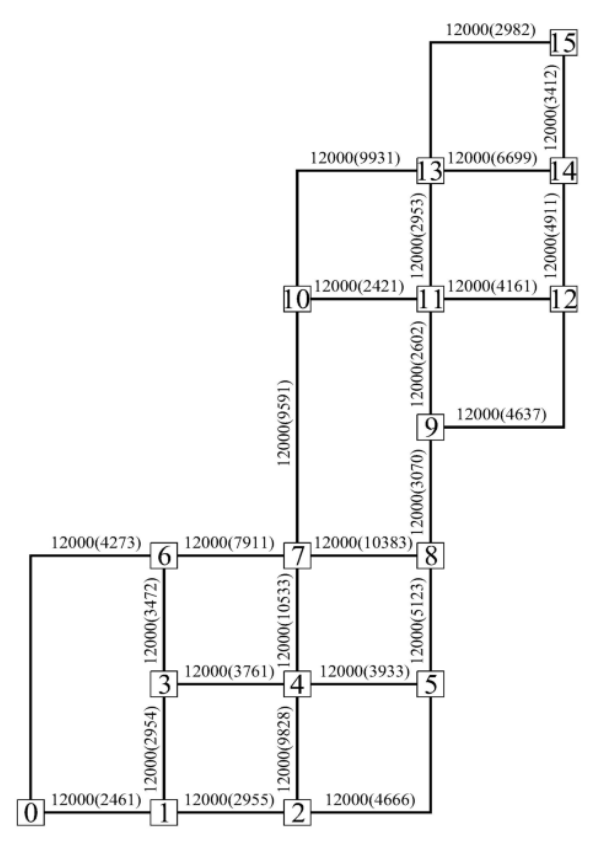

Fig 2. The node number of prediction results

Consider the development of the area, select the line 1-4 for analysis. The network algorithm, line index and passenger flow index are input into the solution model. The iterative process of genetic algorithm is as Figure 3.

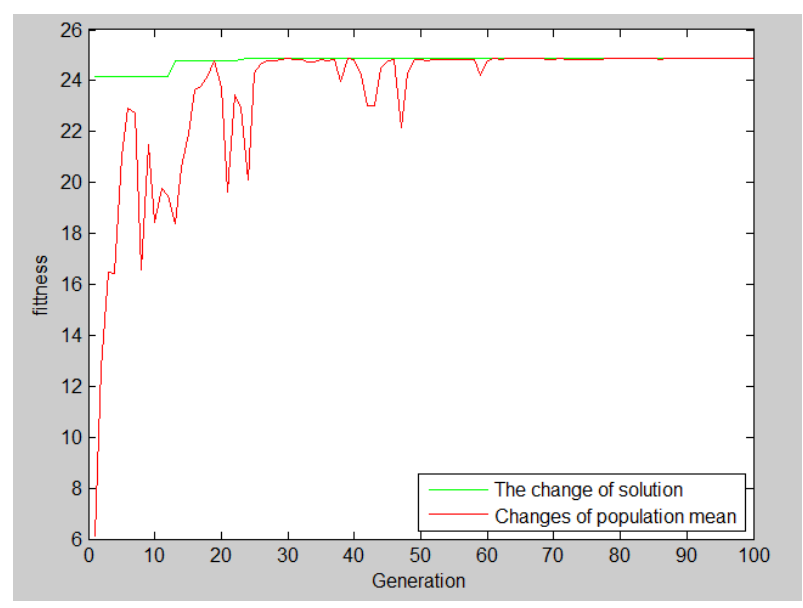

Fig 3. The result of iteration

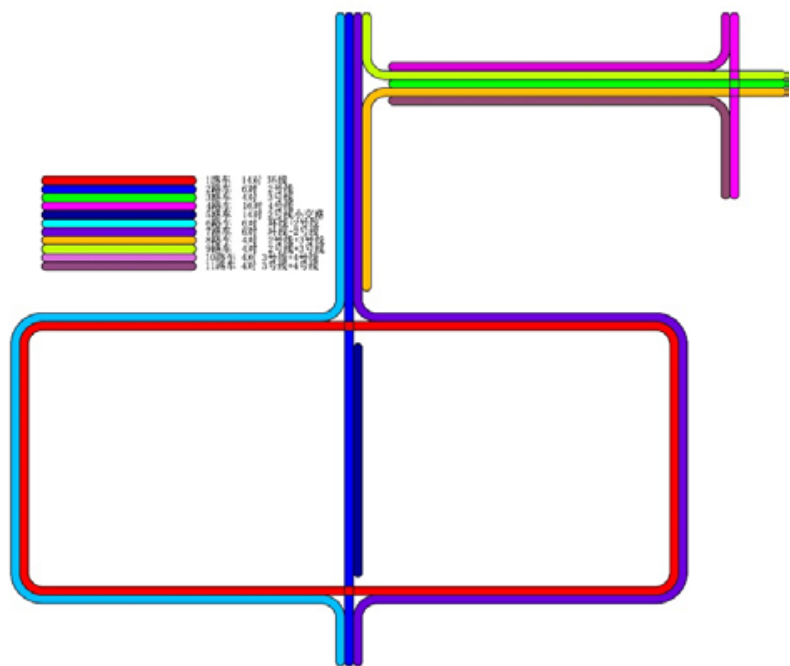

Fig 4. Modern tram operating planning of tramway network

Take theresult into the optimization model,and the maximum value is: $V^{1}=\left\{v_{2}, v_{4}, v_{7}, v_{10}\right\}$; Repeat the above results, the second choice of line results: $V^{2}=\left\{v_{0}, v_{6}, v_{7}, v_{8}\right\} \ldots$ So on and so forth, Get the initial line operation plan as shown in Figure 4. 


\section{Conclusion}

(1) Based on the technical characteristics of urban rail transit and common transit , this paper analyzes the traditional method of network optimization, and puts forward the research method of the optimization of the running line which is suitable for the characteristics of tram. By modeling and solving the optimization method, which concluded that the operating results are in accordance with the parameters and constraints of the model.

(2) In the actual operation process, there are many factors influencing the operation route of the tram, including the development of urban land use, the characteristics of road traffic, the priority control of the intersection signal, the road section and so on, and the adjustment of the theoretical and theoretical results should be adjusted according to local conditions.

\section{Acknowledgments}

This work was financially supported by 863 project (2007AA11Z248) fund.

\section{References}

[1] Standard for classification of urban public transportation[S] . (CJJ/T 114-2007).

[2]Lu Ximing, \& Li Na. 2013. Rational and Sustainable Development of Streetcars[J], Urban Transport of China , 11(4):19-23.

[3] Zhang Zidong. 2013. Streetcar System Planning and Design[J], Urban Transport of China , 11(4):30-38.

[4] CHEN Kuan-min, \& FAN Da-ke, \& MA Chao-qun. 2009. Cal-culation of urban rail transit network scale based on pas-senger distributing centers[J], Journal of Chang 'an Uni-versity(Natural Science Edition), 29(6):78-81.

[5] Herbon, A., \& Hadas, Y. (2015). Determining optimal frequency and vehicle capacity for public transit routes: A generalized newsvendor model. TRANSPORTATION RESEARCH PART B-METHODOLOGICAL, 71, 85-99. doi: 10.1016/j.trb.2014.10.007.

[6] LUO Xiao-qiang, \& CHEN Kuan-min. 2008. Determined mod-els of optimal length for urban rail transit line[J]. Journal of Traffic and Transportation Engineering, 10(3):64-65.

[7] MA Chao-qun,\& WANG Yu-ping.(2010). Method to optimize urban rail transit network based on maximizing the passenger flow[J]. Journal of Chang 'an University(Natural Science Edition), 30(1) : 76-79.

[8] Zheng Liping, \& Hao Zhongxiao. 2003. A revised on the theory for the Genetic Algorithm[J]. Computer Engi-neering and Applications, 21:50-53. 